\title{
Mass poisoning after consumption of a hawksbill turtle, Federated States of Micronesia, 2010
}

\author{
Boris I Pavlin, a Jennie Musto, ${ }^{b}$ Moses Pretrick, ${ }^{c}$ Joannes Sarofalpiy, ${ }^{c}$ Perpetua Sappa, ${ }^{d}$ Siana Shapucy ${ }^{d}$ and \\ Jacobus Kool ${ }^{b}$ \\ Correspondence to Boris I Pavlin (e-mail: pavlinb@wpro.who.int).
}

Background: Marine turtles of all species are capable of being toxic. On 17 October 2010, health authorities in the Federated States of Micronesia were notified of the sudden death of three children and the sickening of approximately 20 other people on Murilo Atoll in Chuuk State. The illnesses were suspected to be the result of mass consumption of a hawksbill turtle (Eretmochelys imbricata). An investigation team was assembled to confirm the cause of the outbreak, describe the epidemiology of cases and provide recommendations for control.

Methods: We conducted chart reviews, interviewed key informants, collected samples for laboratory analysis, performed environmental investigations and conducted a cohort study.

Results: Four children and two adults died in the outbreak and 95 others were sickened; $84 \%$ of those who ate the turtle became ill $(n=101)$. The relative risk for developing illness after consuming the turtle was 11.1 (95\% confidence inteval: 4.8-25.9); there was a dose-dependent relationship between amount of turtle meat consumed and risk of illness. Environmental and epidemiological investigations revealed no alternative explanation for the mass illness. Laboratory testing failed to identify a causative agent.

Conclusion: We concluded that turtle poisoning (also called chelonitoxism) was the cause of the outbreak on Murilo. The range of illness described in this investigation is consistent with previously reported cases of chelonitoxism. This devastating incident highlights the dangers, particularly to children, of consuming turtle meat. Future incidents are certain to occur unless action is taken to alter turtle-eating behaviour in coastal communities throughout the world.

$\mathrm{M}$ arine turtles of all species, particularly hawksbill turtles (Figure 1 ), can contain a variety of toxins including heavy metals (e.g. cadmium and mercury), organic compounds (e.g. pesticides such as chlordane and polychlorinated biphenyls) and biotoxins such as those produced by various blue-green algae on which turtles feed (e.g. lyngbyatoxin A from Lyngbya majuscula). ${ }^{1}$ Intoxication from consumption of marine turtles is called chelonitoxism. Symptoms of chelonitoxism vary according to the toxin and include gastrointestinal symptoms of nausea, vomiting and oro-esophageal ulceration, sometimes followed in severe cases by neurologic signs such as paresis, agitation and coma. ${ }^{1,2}$ Despite these hazards and despite the endangered status of all marine turtle species, these turtles are eaten by coastal communities throughout the world. ${ }^{1}$

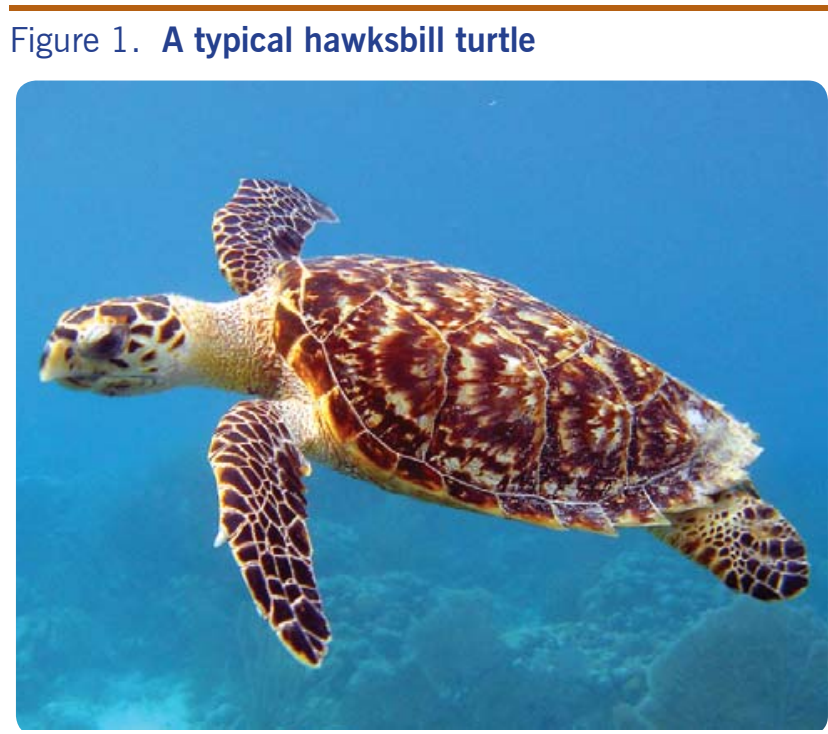

Source: http://commons.wikimedia.org/wiki/File:Hawksbill_Sea_Turtle_ Carey de Concha_(5840602412).jpg.

\footnotetext{
Office of the WHO Representative in Papua New Guinea, Port Moresby, Papua New Guinea.

Division of Pacific Technical Support, World Health Organization, Suva, Fiji.

Department of Health and Social Affairs, Government of the Federated States of Micronesia, Pohnpei, Federated States of Micronesia.

Chuuk State Hospital, Chuuk State Department of Health Services, Chuuk, Federated States of Micronesia.

Submitted: 9 September 2014; Published: 26 January 2015

doi: 10.5365/wpsar.2014.5.3.006
} 
The island nation of the Federated States of Micronesia comprises of 607 islands in the North Pacific Ocean (Figure 2). On Sunday, 17 October 2010, the Federated States of Micronesia Department of Health and Social Affairs and the World Health Organization (WHO) were notified of the sudden death of three children and the sickening of approximately 20 other people on Murilo Atoll, a small, isolated island with a population of approximately 330, located $161 \mathrm{kms}$ from the main population centre of Weno Island in Chuuk State. The illnesses were suspected to be the result of mass consumption of a hawksbill turtle (Eretmochelys imbricata) that had been prepared and served on the afternoon of 15 October. Shortly after the initial report, the deaths of another child and two hospitalized adults were reported; dozens of residents developed sore throats, and several dogs died suddenly.

A response team set up a field hospital on Murilo, and a investigation team was sent to confirm the cause of the outbreak, describe the epidemiology of cases and provide recommendations to prevent additional cases or outbreaks.

\section{METHODS}

\section{Hypothesis generation}

The outbreak investigation team held a community meeting on Murilo on 25 October to explain the purpose of the investigation, gather initial information and answer questions from community members. On the morning of 26 October, the team interviewed all 20 community members who did not eat the turtle but who reported feeling ill to determine whether there was any other threat besides turtle poisoning that could explain the reported illnesses.

Information on all known ill persons was reviewed to determine onset dates and whether there was an ongoing environmental threat or an infectious condition. Key informants from the community were interviewed about foods consumed as part of the turtle feast; presence of unusual environmental conditions such as chemical spills, algal blooms, fish die-offs and changes in reef ecology; and possibility of infectious etiology.

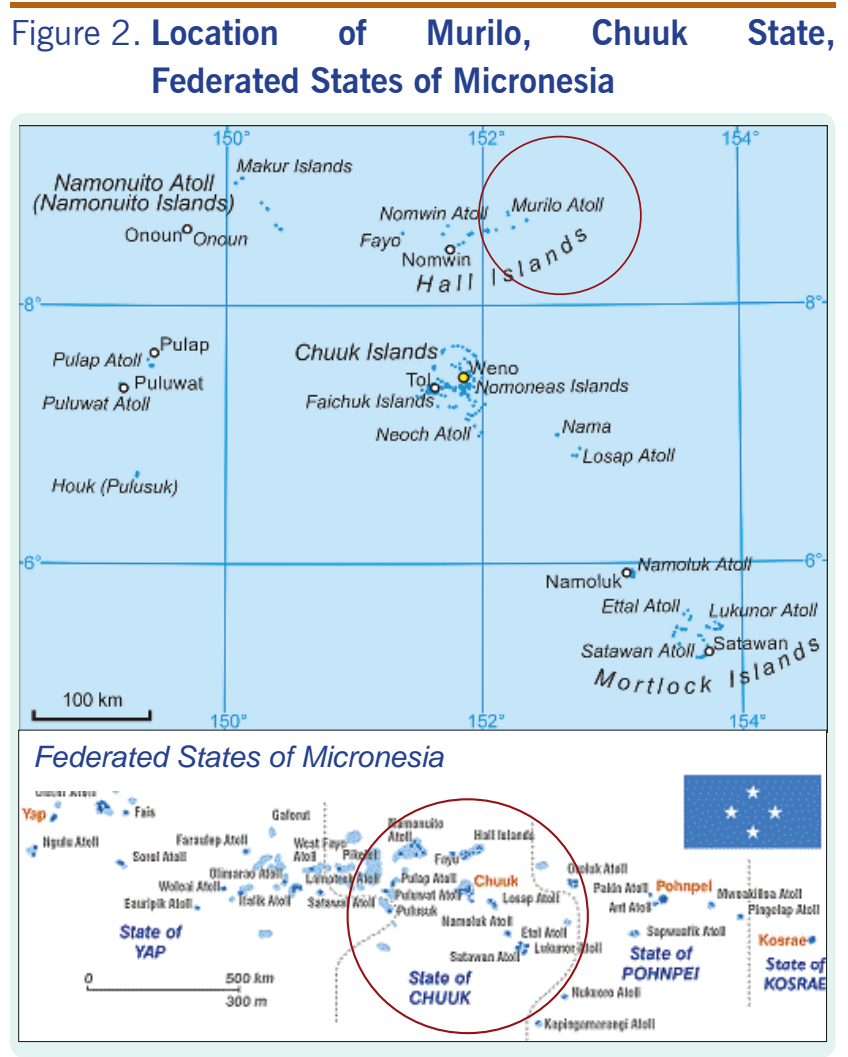

Source: Chuuk State map was reproduced from Wikimedia Commons, the free media repository (http://commons.wikimedia.org/wiki/File:Chuuk.png), and the inset map of the Federated States of Micronesia was reproduced from WHO Division of Pacific Technical Support (http://www.wpro.who. int/southpacific/pacelf/countries/fsm/en/).

\section{Case series}

A case was defined as a resident of Murilo who had sudden death, sore throat, mouth sores or excessive thirst with onset of illness between 15 and 28 October. All cases who had eaten turtle (or were breastfed by mothers who had eaten turtle) were interviewed to determine the amount of turtle consumed, onset of illness and clinical features. Proxy interviews were conducted with the local health assistant and family members of the six deceased cases; the medical charts of the two decedents who had been hospitalised were also reviewed.

\section{Cohort study}

A retrospective cohort study was conducted to determine risk factors for illness using the same case definition as per the case series. All persons present at the turtle feast and their household members were 
Figure 3. Cases of turtle poisoning by date of onset of illness

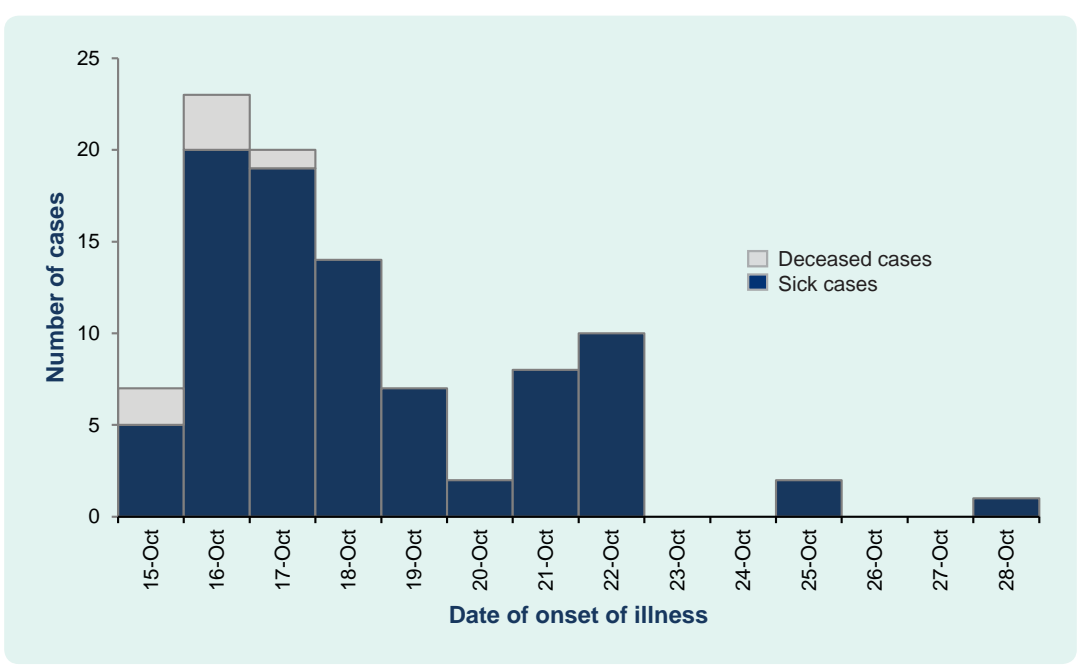

interviewed (or proxy interviewed if deceased) with a standardized questionnaire. This comprised questions on demographics, preparation and consumption of turtle, consumption of other foods and drinks and illness. The quantity of turtle soup consumed was reported as multiples of a $70 \mathrm{~mL}$ jar. Data were analysed using Microsoft Excel 2003, Epilnfo 3.5.1 and STATA 9. Univariate logistic regression was used to examine the relationship between illness and the risk factors of age, sex, village, involvement in turtle preparation and consumption of turtle (no other food items were common to more than a few people). Any risk factors approaching significance $(P<0.2)$ in univariate analysis were retained for multivariate logistic regression. As this research was part of an emergency public health response, no institutional review board consultation was needed.

\section{Environmental investigation}

Members of the investigation team together with an authority from the Chuuk Department of Marine Resources conducted an environmental assessment to look for evidence of contamination. Additionally, every effort was made to secure food samples from the turtle feast for laboratory testing.

\section{Laboratory testing}

Samples of patient sera, turtle scutes (shell plates) and bones, internal organs from a deceased dog and algae were sent to Forensic and Scientific Services, Queensland, Australia. Testing was conducted for the following marine toxins: lyngbyatoxin $A$, debromoaplysiatoxin, okadaic acid, gymnodimine, pectenotoxin 2 and dinophysistoxins.

Ethics approval was not required as this investigation was conducted as part of a public health intervention. Informed consents were obtained from all participants and for deceased cases from their next-of-kin.

\section{RESULTS}

\section{Hypothesis generation}

Of the 20 ill people who did not eat turtle, 10 were diagnosed as influenza-like illness. The remaining 10 were attributed to various other ailments. All cases had a date of illness onset on or before 22 October, with the exception of three outliers (Figure 3 ). Many residents of Murilo reported eating reef fish and other local foods around the time of the turtle feast and they did not become ill.

The turtle was captured alive from the Murilo lagoon using a hook and line (no chemicals) approximately one week before the turtle feast and was apparently healthy. It was kept alive on land, but it died in the early part of the day of the feast, thought to be from heat exhaustion. The turtle was prepared simply by chopping all meat and organs into a single pot for cooking; it was not gutted. The turtle was then boiled in fresh water for two to three hours, after which the contents of the first pot were further chopped and boiled a second time after discarding and replacing the water. Each household 
(including members of the extended family) was given a bowl of the turtle stew. In almost all instances, the stew was consumed immediately after it was served. Each household ate its own variety of accompaniments with the stew (e.g. rice, breadfruit, taro); there were no other common foods served at the feast.

The two adult brothers who died were involved in the butchering and cooking of the turtle. They were reported to have eaten a large amount of turtle at the feast (but not more than several others); it was also reported that the brothers may have eaten large amounts of turtle during cooking and preparation.

Residents reported that sea turtles were consumed on the island approximately twice a month, with most being green sea turtles (Chelonia mydas). There had never been an incident related to turtle poisoning on Murilo before this outbreak. Other hawksbill turtles had been consumed in the past year without incident. Residents were not aware of any hazard associated with consuming sea turtles.

\section{Case series}

Of the 120 people who consumed turtle (or who were breastfed by mothers who ate turtle), 101 ill people met the case definition. Females accounted for $51 \%$ of cases $(n=52)$ and the median age was 17 years (range $=$ 5 weeks to 70 years). The median incubation period from turtle consumption to illness was two days (range $=$ 2 hours to 13 days) (Figure 3). Medical attention was sought by $37 \%$ of cases $(n=37)$. Interviews were conducted 12 days after the exposure; only 27 of the 101 ill people had recovered at the time of interview. All surviving patients have reportedly since recovered.

Sore throat was the most frequently reported symptom, occurring in $84 \%$ of cases for whom responses were available $(n=76)$. Other commonly reported symptoms included mouth pain $(78 \%, n=72)$, thirst $(71 \%, n=66)$ and burning throat $(54 \%, n=47$; Table 1).

Eight cases were breastfed infants whose mothers had consumed turtle, although two infants also consumed turtle themselves.

Several patients whose mouths were examined had pale yellow exudative lesions of the oropharynx and
Table 1. Number and proportion of reported symptoms in cases, Murilo, 2010*

\begin{tabular}{lccc}
\hline \multicolumn{1}{c}{ Symptom } & Number & Respondents & Percentage (\%) \\
\hline Sore throat & 76 & 90 & 84 \\
Mouth pain & 72 & 92 & 78 \\
Thirst & 66 & 93 & 71 \\
Burning throat & 47 & 87 & 54 \\
Fever & 40 & 94 & 43 \\
Cough & 35 & 93 & 38 \\
Vomiting & 29 & 94 & 31 \\
Abdominal pain & 26 & 93 & 28 \\
Epigastric pain & 22 & 87 & 25 \\
Constipation & 20 & 94 & 21 \\
\hline
\end{tabular}

* Number of respondents varies as not all patients responded to all questions because several of the patients were infants.

tongue. This was ubiquitous among those who had eaten turtle and had presented to the field hospital.

\section{Fatal cases}

Case 1 was a previously healthy five-year-old girl who consumed turtle meat at approximately 16:00 on 15 October and then vomited once approximately 24 hours after the meal. Her only other reported symptom was thirst; however, she refused all oral liquids when offered. Within 36 hours after consumption, on the morning of 17 October, she developed seizures and died shortly afterwards.

Case 2 was a previously healthy two-year-old girl and the sister of Case 1 who died within minutes of her sibling. The mother reported that the child woke irritable, distressed and with abdominal pain at approximately 03:00 on 16 October, was settled after breastfeeding but died shortly after. Case 2 consumed a small amount of turtle meat but was breastfed by her mother within three hours after she had eaten turtle.

Case 3 was a previously healthy two-year-old boy who did not consume turtle but was breastfed by his mother after she consumed large amounts of turtle. Case 3 had two episodes of diarrhoea on the night of 16 October and another in the early morning of 17 October. He died shortly thereafter.

Case 4 was a previously healthy 21-year-old man who consumed turtle at approximately 16:00 on 15 October. Approximately two hours later he experienced 
Table 2. Dose-response of odds of illness for amounts of turtle consumed, Murilo, 2010

\begin{tabular}{cccc}
\hline \multirow{2}{*}{ Dose* $^{*}$ Odds ratio } & \multicolumn{2}{c}{$95 \%$ confidence interval } \\
& & Lower & Upper \\
\hline None & 1.00 (Reference) & \\
$<1$ & 32.0 & 9.4 & 108.7 \\
1 & 61.0 & 10.4 & 358.4 \\
2 & 122.0 & 12.9 & 1156.0 \\
3 & 146.4 & 15.6 & 1367.5 \\
$4+$ & 77.3 & 22.0 & 270.8 \\
Chi-square $=111.75$ & & & \\
$p$-value $<0.0001$ & & & \\
\hline
\end{tabular}

* 1 dose was a $70 \mathrm{~mL}$ cup. This table does not include five cases who were exclusively breastfed and 11 cases where the amount consumed is unknown.

nausea but did not vomit. The following evening, he vomited more than 10 times and then eventually went to sleep. The next morning he felt better and went fishing. While at sea he developed haematemesis and was evacuated to Chuuk State Hospital on Weno Island by a nearby supply ship. On the boat he became restless and disoriented and did not recognize his relatives. Ambulance drivers on Weno noted the case had an unusual smell unlike anything they had experienced. At the hospital he was agitated and combative and required sedation. He was placed in the intensive care unit and administered intravenous fluids: mannitol for presumed ciguatera intoxication and dexamethasone for presumed cerebral oedema. A neurologic exam found the patient was profoundly lethargic with pinpoint pupils and hyperreflexia in all extremities. Vital signs were within normal ranges except for an elevated respiratory rate. Laboratory values were unremarkable except for mild neutrophilia. Radiography was unremarkable.

Over the following two days, the patient remained profoundly lethargic and developed sustained clonus and an upward plantar reflex. His chemistries were notable for markedly elevated aminotransferases, amylase and creatinine. The patient remained in this state until 21 October when he developed respiratory distress requiring intubation and mechanical ventilation. He was removed from the ventilator at the request of his family in the early morning of 22 October and died at 04:00.
Case 5 was the brother of Case 4, a previously healthy 22-year-old man who consumed turtle at approximately 16:00 on 15 October. Three hours later he felt nauseated, did not vomit but reported thirst. On the following evening he vomited several times. On the third day he was noted to have headache, weakness, fever, disorientation and agitation. $\mathrm{He}$ was taken to the hospital intensive care unit on the morning of 19 October and placed on intravenous fluids: mannitol for presumed ciguatera intoxication and dexamethasone for presumed cerebral oedema. He was restless and disoriented but otherwise unremarkable. Initial radiography and laboratory investigation were also unremarkable.

Over the course of the evening the patient became agitated and combative and required sedation. On the evening of 21 October the patient developed severe respiratory distress, and he died at 01:00 on 22 October.

Case 6 was a previously healthy four-year-old boy who ate turtle on the evening of 15 October. He vomited on the evenings of 16 and 17 October, then appeared well until he reported a sore throat on 21 October. In the early morning of 22 October he vomited once, collapsed and died.

For all decedent cases, except for Case 4 whose serum was sent for laboratory analysis, no samples were taken and autopsies were not performed.

\section{Cohort study}

The cohort study included 186 residents of Murilo present at the turtle feast and their household members. Consumption of turtle stew had an attack rate of $84 \%$ (101/120). The relative risk of developing illness after consuming turtle stew in univariate analysis was 11.1 (95\% confidence interval: 4.8-25.9). In multivariate analysis, consumption of turtle was the only risk factor associated with illness; village and involvement in the preparation of turtle were not (data not shown).

There was a statistically significant dose-response relationship between the amount of turtle consumed and the odds of developing illness (Table 2) with the 
Figure 4. Abundant algae presumed to be Lyngbya majuscula, also known as "Mermaid's Hair"

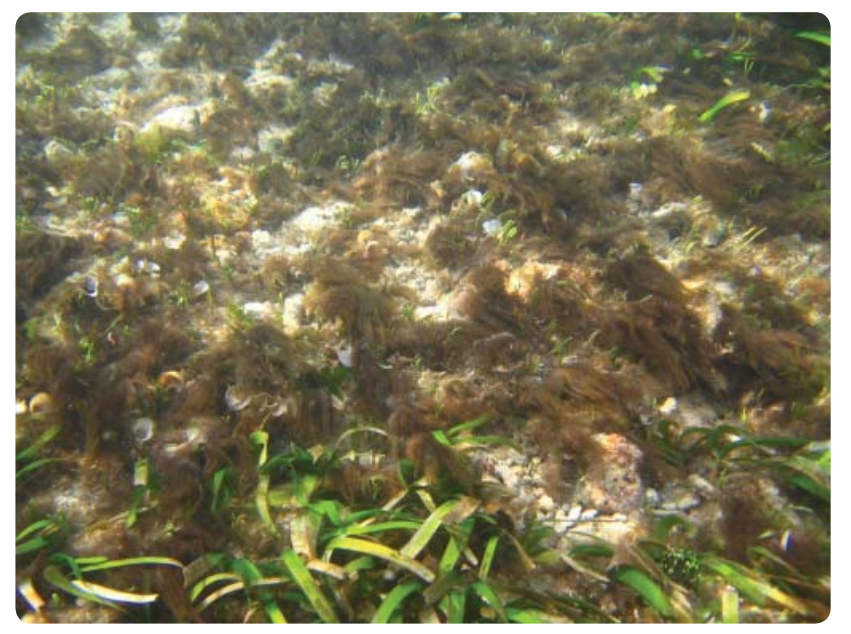

exception of the last category (four or greater). When stratified by age under 10 versus 10 and above, the dose response was similar (data not shown).

\section{Environmental investigation}

No obvious evidence of acute environmental contamination was found. The reef surrounding Murilo was in extremely poor condition, consisting of predominantly dead coral and large amounts of algae presumed to be Lyngbya majuscula (Figure 4).

No leftover turtle meat was found - the community consumed most of the turtle and discarded the remainder into the sea. However, the scutes and several bones were recovered. These were sent for laboratory analysis.

At least six dogs were reported to have died suddenly, presumably after eating turtle leftovers. All but two of these dogs were subsequently discarded or consumed (there were no reports of illness after consuming dogs). Of the remaining two, one was completely decomposed; the other, not completely decomposed, was dissected and stomach contents were sent for laboratory analysis.

\section{Laboratory testing}

All samples tested were below the limit of detection for all toxins tested.

\section{DISCUSSION}

This investigation shows strong epidemiologic evidence that chelonitoxism was the cause of mass illness on Murilo. The convincing dose-response relationship particularly supports that turtle was the cause of illness. We suspect that the dose-response failure of those who reported eating more than four servings of turtle was due to consumption of less turtle stew than reported.

Children are expected to be more severely affected by chelonitoxism due to their lower body mass and more sensitive immune systems. ${ }^{1}$ Therefore the death of several children in this outbreak was not unexpected. It is not clear why one child (Case 6) reported a nonserious illness only to die suddenly several days later. It is also not clear why the two adult males developed serious disease and died, although there was some evidence that they may have eaten larger amounts of turtle than the others before the feast. That they alone may have eaten raw turtle during preparation is likely not relevant as most chelonitoxins are heat-stable. ${ }^{3}$

Several infants developed illness without eating turtle but had been breastfed from a mother who ate turtle. Passage of chelonitoxins through breast milk has been documented. ${ }^{3}$ The toxins are thought to be virtually eliminated from the body within a few days, posing no ongoing threat for breastfeeding infants in Murilo. There is no other known transmission of these toxins from person to person.

The range of illness described in this investigation, from relatively mild mucus membrane irritation to coma and death, is consistent with previously reported cases of chelonitoxism. ${ }^{2}$ Despite intensive care, patients may succumb to the effects of the poison as there is no specific treatment for chelonitoxism. One possible toxin that may explain the widespread illness reported during this investigation is lyngbyatoxin $A$, the major toxin produced by Lyngbya majuscula. It has a direct inflammatory effect on tissue, ${ }^{4}$ which my have caused the sore throats, mouth pain and oral ulcers. Although there is little evidence of neurotoxicity of lyngbyatoxin, it is possible that large quantities may account for the neurologic toxicity seen in the fatal cases and as documented in other reports of severe chelonitoxism. ${ }^{2}$ 
Lyngbyatoxin A has been isolated from a green sea turtle (Chelonia mydas) associated with fatal chelonitoxism ${ }^{5}$ but not a hawksbill turtle. Although hawksbill turtles are carnivores, they also feed on sea grasses and algae, ${ }^{6}$ so algal toxins cannot be ruled out. The clinical picture of chelonitoxism caused by Chelonia mydas is the same as that caused by Eretmochelys imbricata, ${ }^{7,8}$ hence the toxin could have been either of these.

In this study, all human, animal and algal samples were negative for all toxins tested. Chelonitoxins have not been found previously in patient sera and only have been isolated from turtle meat, ${ }^{5}$ not scutes or bones, which were the only turtle tissues available here. Although the algae tested, which appeared to be Lyngbya majuscula, did not contain toxins, we cannot rule out the possibility that lyngbyatoxin was the etiology, as the turtle may have grazed from a different algal site. Alternatively, another toxin that was not tested for may have been the cause. It is possible that failure to detect toxins resulted from inadequate sampling or incomplete testing rather than true absence of toxins in the consumed turtle.

Hawksbill turtles have routinely been eaten on Murilo, yet there is no history of previous poisoning related to turtle consumption. It is not clear why the turtle eaten in this outbreak was toxic; however, most sea turtle species, particularly hawksbill turtles, can be poisonous if consumed. ${ }^{3}$ There is no reliable way to determine which individual turtles are poisonous, but it is more likely if they have eaten foods that may contain high levels of toxins. Foods high in toxins include bluegreen algae such as Lyngbya majuscula and also a variety of sponges and other marine organisms. Turtle eggs are also known to have high levels of a variety of toxins. ${ }^{1}$ There have been reports of other turtle poisonings in the Federated States of Micronesia including on Sapwuahfik, Pohnpei State, in April 2010 (D Buden, personal communication) and two previous incidents (including three deaths) on the same island in $1997 .^{9}$

As any turtle or turtle eggs may be toxic (i.e. Murilo is not unique), we recommended the cessation of consumption of turtle products throughout the Federated States of Micronesia. Even though sea turtles are relatively abundant in Chuuk, they are endangered throughout the world (hawksbill turtles are critically endangered); ${ }^{10}$ therefore, efforts to protect human health by reducing turtle capture have the added benefit of helping to conserve these rapidly disappearing species.
The devastating incident on Murilo highlights the dangers, particularly to children, of consuming turtle meat. Future incidents are certain to occur unless action is taken to alter turtle-eating behaviour in coastal communities throughout the world. Since all turtles and their eggs are capable of being toxic, we strongly recommend ceasing consumption of any turtles or their eggs to protect public health.

\section{Funding}

None.

\section{Conflicts of interest}

None declared.

\section{Acknowledgements}

We would like to acknowledge the assistance of the community of Murilo, the Chuuk Police Department, Department of Health Services, and Department of Marine Resources in our investigation. Particular thanks goes to Ismael Shapucy, Kachusy Farek, Pastor Mesenty, Dave Williander, Tafson Menesio, Riten Billias, Curtis Sos, Alex Narruhn and Benty Rickson. We also thank Forensic and Scientific Services, Queensland, Australia, for providing laboratory testing of samples. Finally, we acknowledge the clinical care and data provided by the late Herliep Nowell.

\section{References:}

1. Aguirre AA et al. Hazards associated with the consumption of sea turtle meat and eggs: A review for health care workers and the general public. EcoHealth, 2006, 3:141-153. doi:10.1007/ s10393-006-0032-x

2. Fussy $A$ et al. Chelonitoxism: new case reports in French Polynesia and review of the literature. Toxicon, 2007, 49:827-832. doi:10.1016/j.toxicon.2006.12.002 pmid:17250862

3. Limpus CJ. Sea turtles. In: Covacevich J, editor. Toxic Plants and Animals: A Guide for Australia. Brisbane, Queensland Museum, 1987:189-194.

4. Osborne NJT, Webb PM, Shaw GR. The toxins of Lyngbya majuscula and their human and ecological health effects. Environment International, 2001, 27:381-392. doi:10.1016/ S0160-4120(01)00098-8 pmid:11757852

5. Yasumoto T. Fish poisoning due to toxins of microalgal origins in the Pacific. Toxicon, 1998, 36:1515-1518. doi:10.1016/ S0041-0101(98)00142-1 pmid:9792166

6. Limpus CJ. Hawksbill turtle, Eretmochelys imbricata (Linnaeus). In: Fein L, editor. A biological review of Australian marine turtle species. Brisbane, Queensland Environmental Protection Agency, 2009: 1-53. 
7. Champetier de Ribes $\mathrm{G}$ et al. Un problème de santé réémergent à Madagascar: les intoxications collectives par consommation d'animaux marins. Aspects épidémiologiques, cliniques et toxicologiques des épisodes notifiés de janvier 1993 à janvier 1998 [In French]. Archives de I'Institut Pasteur de Madagascar, 1998, 64:71-76.

8. Champetier de Ribes $\mathrm{G}$ et al. Intoxications par animaux marins vénéneux à Madagascar (ichtyosarcotoxisme et chélonitoxisme): données épidémiologiques récentes [In French]. Bulletin de la Société de Pathologie Exotique, 2007, 90:268-272.

9. Buden DW. The reptiles of Sapwuahfik Atoll, Federated States of Micronesia. Micronesia, 2000, 32:245-56.

10. IUCN Red List of Threatened Species. Version 2014.3. Cambridge, United Kingdom, International Union of Conservation of Nature and Natual Resources, 2014 (http://www.iucnredlist. org, accessed 4 December 2014). 